\title{
Атомистическое моделирование решеточных свойств SnSe
}

\author{
(C) А.Н. Филанович ${ }^{1}$, Ю.В. Лысогорский ${ }^{2}$, А.А. Повзнер ${ }^{1}$ \\ ${ }^{1}$ Уральский фредеральный университет им. первого Президента России Б.Н. Ельцина, \\ 620002 Екатеринбург, Россия \\ ${ }^{2}$ ICAMS, Ruhr-Universitat Bochum, \\ 44801 Bochum, Germany \\ E-mail: a.n.filanovich@urfu.ru
}

Поступила в Редакцию 12 августа 2021 г.

В окончательной редакции 28 августа 2021 г.

Принята к публикации 28 августа 2021 г.

Выполнен ряд $a b$ initio расчетов энергии основного состояния как функции объема, упругих свойств и фононных спектров селенида олова в его различных кристаллических модификациях. На основе полученного массива данных построен потенциал межатомного взаимодействия $\mathrm{SnSe}$ с использованием метода атомного кластерного разложения (atomic cluster expansion - ACE). С использованием потенциала исследуются температурные зависимости тепловых и упругих свойств $\mathrm{SnSe}$ в рамках квазигармонического приближения.

Ключевые слова: термоэлектрики, решеточный ангармонизм, тепловые свойства, упругие свойства, селенид олова, атомистическое моделирование, потенциал межатомного взаимодействия.

DOI: 10.21883/FTP.2021.12.51698.13

\section{1. Введение}

Селенид олова является перспективным термоэлектрическим материалом со значением добротности $Z T$ вплоть до $\sim 2.6$ при температуре $923 \mathrm{~K}[1]$. В составе $\mathrm{SnSe}$ отсутствуют токсичные элементы, что усиливает интерес к использованию данного материала в области „зеленой“ энергетики. Столь высокое значение ZT обусловлено прежде всего низкой решеточной теплопроводностью $\mathrm{SnSe}$, которая в свою очередь объясняется сильным решеточным ангармонизмом. При атмосферном давлении и нуле температур $\mathrm{SnSe}$ обладает орторомбической структурой с группой симметрии Pnma, где атомы $\mathrm{Sn}$ и $\mathrm{Se}$ образуют кристаллические слои, состоящие из двух плоскостей зигзагообразных цепочек $\mathrm{Sn}-\mathrm{Se}$ [2]. Внутри слоев атомы связаны прочными гетерополярными связями, в то время как между слоев слабыми силами Ван-дер-Ваальса и дальнодействующим кулоновским притяжением. В области $800 \mathrm{~K}$ происходит фазовый переход в другую слоистую орторомбическую структуру с группой симметрии $\mathrm{Cmcm}$.

Учитывая то, что выдающиеся термоэлектрические свойства $\mathrm{SnSe}$ во многом обусловлены ангармоничностью его связей и решеточных колебаний, важным представляется понимание фононной подсистемы $\mathrm{SnSe}$ и ее влияния на решеточные свойства. Несмотря на достаточно большое количество работ, в которых исследуется динамика решетки $\mathrm{SnSe}$ (см., например, [3-12]), его решеточные свойства изучены недостаточно. Одной из загадок является неоднозначность величины параметра Грюнайзена Г, который известен как основная характеристика фононного ангармонизма. Так, исходя из рассчитанной зависимости фононных частот от объема [8], а также из измерений скорости ультразвука при $300 \mathrm{~K}$ [11], следует, что параметр Грюнайзена принимает значения в районе $3.13-3.15$, что свойственно веществам с силь- ной ангармоничностью решеточных колебаний. С другой стороны, исходя из значений термодинамических свойств и известного соотношения $\Gamma=\beta B_{T} V / C_{V}$, где $\beta$ - объемный коэффициент теплового расширения (ОКТР), $B_{T}$ - изотермический модуль всестороннего сжатия, $V$ и $C_{V}$ - молярные значения объема и теплоемкости при постоянном объеме, следует, что при $T=300 \mathrm{~K} \Gamma$ селенида олова составляет всего $\sim 0.66$.

Симуляции на основе классической молекулярной динамики являются одним из способов вычисления термодинамических свойств с учетом ангармонических эффектов. Однако для осуществления таких симуляций необходим потенциал межатомного взаимодействия, достоверно описывающий свойства материала. В настоящей работе мы строим потенциал $\mathrm{SnSe}$ на основе атомного кластерного разложения (Atomic Cluster Expansion, ACE) [13]. Предварительно выполнен ряд первопринципных расчетов для различных структурных модификаций $\mathrm{SnSe}$ с помощью теории функционала плотности (Density Functional Theory - DFT), что позволило сформировать необходимую базу данных для фиттинга потенциала. Далее на основе полученного потенциала мы рассчитываем температурные зависимости ряда решеточных свойств $\mathrm{SnSe}$ в рамках квазигармонического приближения и сравниваем их с доступными экспериментальными данными.

\section{2. Методика исследования}

\section{1. Потенциал межатомного взаимодействия на основе атомного кластерного разложения (ACE)}

Межатомные потенциалы являются упрощенными моделями межатомного взаимодействия и широко используются в компьютерном моделировании материалов, в 
частности, для симуляций, которые являются вычислительно слишком затратными при применении DFT, использование при этом межатомных потенциалов позволяет ускорять расчеты вплоть до нескольких тысяч раз.

Атомное кластерное разложение (atomic cluster expansion, ACE) - это широкий класс межатомных потенциалов, основанный на обобщенном полиномиальном разложении энергии отдельных атомов на многоатомные базисные функции $[13,14]$. Потенциальная энергия отдельного атома $E_{i}$ может быть представлена в виде общего вида нелинейной функции от атомных плотностей $\phi_{i}^{(p)}$. В нашем случае мы использовали выражение вида

$$
E_{i}=\phi_{i}^{(1)}-\sqrt{\phi_{i}^{(2)}},
$$

где атомная плотность представляет собой линейную комбинацию

$$
\phi_{i}^{(p)}=\sum_{\boldsymbol{v}} c_{\boldsymbol{v}}^{(p)} B_{i v}
$$

Здесь $\boldsymbol{v}=(\boldsymbol{\mu} \mathbf{n I L})-$ сгруппированные коэффициенты, а $c_{\boldsymbol{v}}^{(p)}$ - подбираемые коэффициенты разложения перед В-базисными функциями

$$
B_{i \mu \mathrm{nlL}}=\sum_{m}\left({ }_{\mathbf{L M}}^{\mathrm{Im}} L_{R}=0\right) A_{i \mu \mathrm{nlm}}
$$

где $\left(\operatorname{lm}_{\mathrm{LM}}^{\mathrm{m}} L_{R}=0\right)$ - обобщенные коэффициента КлебшаГордана, используемые для обеспечения вращательной инвариантности. В свою очередь многочастичные базисные функции

$$
A_{i \mu \mathrm{nlm}}=\prod_{t=1}^{v} A_{i \mu_{t} n_{t} l_{t} m_{t}}
$$

являются произведением атомных базисов

$$
A_{i \mu n l m}=\sum_{j} \delta_{\mu \mu_{j}} \varphi_{\mu_{i} \mu_{j} \operatorname{nlm}}\left(\mathbf{r}_{j i}\right),
$$

где суммирование происходит по всем соседним атомам $j$ в пределах сферы с радиусом $r_{\text {cut }}$, называемым радиусом отсечки, а одночастичные функции $\varphi$ выражаются как произведение радиальных функций, зависящих только от расстояния до соседнего атома и сферических гармоник, зависящих от направления до него:

$$
\varphi_{\mu_{i} \mu_{j} n \operatorname{lm}}=R_{n l}^{\mu_{i} \mu_{j}}\left(r_{j i}\right) Y_{\operatorname{lm}}\left(\hat{\mathbf{r}}_{j i}\right) .
$$

Для дополнительных подробностей мы рекомендуем читателю обратиться к работам $[13,14]$.

\section{2. Расчеты термодинамических свойств на основе АCE-потенциала}

Как уже указывалось выше, АCЕ-потенциал позволяет осуществлять расчеты, в том числе сил, действующих на атомы при их смещениях из положения равновесия, значительно быстрее по сравнению с расчетами на основе DFT. Это позволяет осуществлять быстрые расчеты фононного спектра при различных значениях объема с целью проведения симуляций тепловых и упругих свойств в квазигармоническом приближении (QНА). Фононная составляющая свободной энергии Гельмгольца записывается с использованием известной формулы

$$
F_{\mathrm{ph}}(V, T)=\int_{0}^{\infty}\left[\frac{h v}{2}+k_{\mathrm{B}} T \ln \left(1-e^{\frac{-h v}{\mathrm{k}_{\mathrm{B}} T}}\right)\right] g(v) d v,
$$

где $v$ - фононная частота, $g(v)$ - зависящая от объема плотность фононных состояний, $T-$ температура, $h, k_{\mathrm{B}}$ - постоянные Планка и Больцмана соответственно. Прибавляя к данной величине энергию основного состояния кристалла $E(V)$, и минимизируя получаемую свободную энергию по объему, можно определить равновесный объем при желаемом значении температуры. Далее, численно дифференцируя объем по температуре, можно рассчитать объемный коэффициент теплового расширения (ОКТР), а через вторые производные от свободной энергии по объему и температуре - модуль всестороннего сжатия (МВC) и теплоемкость соответственно.

\section{3. Детали ab initio расчетов}

С целью построения потенциала межатомного взаимодействия, достоверно описывающего свойства различных фаз $\mathrm{SnSe}$, был выполнен ряд DFT-расчетов для основных фаз $\mathrm{SnSe}$ при атмосферном давлении Pnma и Cmcm, фазы $F m 3 m$, являющейся, согласно некоторым исследованиям [7], стабильной при высоких температурах, а также некоторых других орторомбических „протоструктур“ $\mathrm{SnSe}$. Для каждой фазы была выполнена геометрическая оптимизация кристаллической структуры (включая как оптимизацию векторов решетки, так и атомных позиций), после чего рассчитана зависимость энергии основного состояния как функция объема $E(V)$ (уравнение состояния) для идеальной примитивной ячейки, а также для ряда искаженных ячеек (см. разд. 3) с целью моделирования структуры $\mathrm{SnSe}$ при конечных температурах. Для фаз Рnma и Cmcm дополнительно были заложены значения энергий и сил для деформированных структур, получаемых при расчете упругих постоянных с помощью метода энергиидеформации [15], фононного спектра методом конечных смещений (подробнее в разд. 3.2), а также расчетов $E(V)$ для искаженных суперъячеек $3 \times 1 \times 1,1 \times 3 \times 1$ и $1 \times 1 \times 3$. Наконец, с целью корректного учета анизотропного теплового расширения, наблюдаемого для Pnта-фазы [8], набор данных по Рnma был дополнен расчетами $E(V)$ для анизотропно деформированных элементарных ячеек. На основе перечисленных данных была получена первоначальная версия потенциала, которая использовалась при выполнении молекулярнодинамических симуляций с помощью программного пакета LAMMPS $[16,17]$ с целью получения „снимков“ кристаллических структур Pnma, Cmcm и Fm3m фаз 
Таблица 1. Параметры кристаллической структуры Pnma и Cmcm фаз $\mathrm{SnSe}$

\begin{tabular}{c|c|c|c|c|c|c|c|c}
\hline & \multicolumn{4}{|c|}{ Pnma } & \multicolumn{4}{c}{$C m c m$} \\
\cline { 2 - 9 } & $a, \AA$ & $b, \AA$ & $c, \AA$ & $V, \AA^{3}$ & $a, \AA$ & $b, \AA$ & $c, \AA$ & $V, \AA^{3}$ \\
\hline DFT & 11.773 & 4.209 & 4.556 & 225.76 & 4.291 & 12.006 & 4.300 & 221.58 \\
ACE & 11.863 & 4.198 & 4.532 & 225.73 & 4.293 & 12.096 & 4.287 & 222.67 \\
$\exp$ & 11.500 & 4.154 & 4.446 & 212.39 & 4.410 & 11.705 & 4.318 & 222.89
\end{tabular}

при конечных температурах. Данные этих структур были заложены при построении окончательной версии потенциала, которая в итоге создавалась на основе данных 725 структур, включающих в сумме 16184 атомов, с энергиями в интервале от -4.111 до -2.018 эВ/атом.

При выполнении DFT-расчетов использовался программный пакет VASP [18,19], псевдопотенциал электрон-ионного взаимодействия на основе метода проекционных присоединенных волн (PAW) [20], обменно-корреляционный функционал был выбран в форме GGA PBE [21]. Энергия обрезания плоских волн составляла 500 эВ, шаг сетки в $k$-пространстве $0.121 / \AA ̊$, что, например, для элементарных ячеек Pnma и Cmcm означает $k$-решетки $5 \times 13 \times 12$ и $11 \times 4 \times 12$ соответственно. При выполнении релаксации структур расчет считался сошедшимся, если изменение абсолютного значения каждой из сил, действующих на атомы, не превышало 0.01 эВ/А. Для организации множества DFT-расчетов и формирования набора данных использовались программные пакеты Pyiron [22] и ASE [23].

\section{3. Результаты и обсуждение}

\section{1. Кристаллические структуры}

Расчеты для элементарных ячеек базировались на основе экспериментальных данных для Pnma [24], Cmcm [25] и Fm3m [26] фаз соответственно. Данные по орторомбическим „протоструктурам“ с группами симметрии Cmme, Cmmm и Pbcm были заимствованы из открытого репозитария Materials Project [27]. При генерации деформированных структур атомные позиции идеальной структуры изменялись случайным образом в интервале $\pm 0.2 \AA$, а компоненты векторов трансляции ячейки увеличивались/уменьшались на случайное значение в интервале $6 \%$. Для генерации случайных значений использовалось стандартное распределение Гаусса.

\section{2. Валидация АСЕ-потенциала на свойствах В основном состоянии}

При фиттинге потенциала значение радиуса отсечки было принято равным $7.5 \AA$ А. Итоговый потенциал содержит в себе 3222 функции с 7344 параметрами и обеспечивает среднеквадратическое отклонение 3.3 (мэВ) для предсказываемых значений энергии и $36($ мэВ/А) для предсказываемых значений сил. В табл. 1 приведены параметры решетки и объемы элементарных Pпma и Cmcm ячеек, полученных на основе ACE-потенциала и с помощью DFT-расчетов в сопоставлении с имеющимися экспериментальными данными [24] для Pnma и [25] для $\mathrm{Cmcm}$.

Можно видеть, что значения, предсказываемые АCEпотенциалом, практически в точности повторяют референтные данные DFT-расчетов, которые также находятся в хорошем согласии с экспериментальными данными: отличие в значениях параметров решетки не превышает 3\%. DFT-расчеты более значительно переоценивают объем ячейки Рпта-структуры по сравнению с Cmcm-структурой, при этом примечательно, что в случае последней, объем, предсказываемый ACEпотенциалом, оказывается ближе к экспериментальному значению по сравнению с результатом DFT.

При вычислении упругих констант энергия для каждого вида деформации рассчитывалась при 7 значениях относительной деформации в интервале от -0.03 до 0.03 c релаксацией атомных позиций в каждой деформированной структуре. Для вычисления упругих модулей и коэффициента Пуассона $v$ использовалось приближение Фойгта-Рейсса-Хилла (VRH) [28]. В табл. 2,a) приведено сопоставление результатов расчета упругих констант и модулей Pnта-фазы SnSe с помощью DFT и АCЕ-потенциала в сопоставлении с экспериментальными данными [29] и результатами расчета [8]. Видно, что, как и в случае параметров кристаллической структуры, результаты расчета на основе DFT и ACEпотенциала очень близки. При сравнении их с данными эксперимента наблюдаем хорошее согласие по постоянным $C_{11}, C_{22}$ и $C_{33}$, и расхождение по $C_{44}$, $C_{55}$ и $C_{66}$. Следует отметить, однако, что результаты расчета этих упругих констант авторами [8] также не очень хорошо согласуются с экспериментом. Это можно объяснить их малыми значениями, когда погрешность расчета может оказаться сопоставима по величине со значением константы. Насколько известно авторам, для Cmcm-фазы отсутствуют экспериментальные данные по упругим свойствам, поэтому в табл. 2,b) результаты наших расчетов сравниваются с результатами расчетов [30].

В целом наблюдается хорошее согласие как между данными DFT- и ACE-расчетов, так и между ними и результатами [30]. Cmcm-структура $\mathrm{SnSe}$ динамически неустойчива при нулевой температуре, что объясняет близкие к нулю значения упругих констант $C_{44}$ и $C_{66}$. 
Таблица 2. Упругие постоянные и модули $\mathrm{SnSe}$

\begin{tabular}{|c|c|c|c|c|c|c|c|c|c|c|}
\hline & $\begin{array}{l}C_{11}, \\
\text { GPa }\end{array}$ & $\begin{array}{l}C_{22}, \\
\text { GPa }\end{array}$ & $\begin{array}{l}C_{33}, \\
\mathrm{GPa}\end{array}$ & $\begin{array}{l}C_{44}, \\
\mathrm{GPa}\end{array}$ & $\begin{array}{l}C_{55}, \\
\mathrm{GPa}\end{array}$ & $\begin{array}{l}C_{66} \\
\mathrm{GPa}\end{array}$ & $\begin{array}{l}B_{\mathrm{H}}, \\
\mathrm{GPa}\end{array}$ & $\begin{array}{l}E_{\mathrm{H}}, \\
\mathrm{GPa}\end{array}$ & $\begin{array}{l}G_{\mathrm{H}}, \\
\mathrm{GPa}\end{array}$ & $v_{\mathrm{H}}$ \\
\hline & \multicolumn{10}{|c|}{ a) Рnпа-фаза } \\
\hline DFT & 43.2 & 64.7 & 28.2 & 28.7 & 14.3 & 11.5 & 24.1 & 36.8 & 14.8 & 0.25 \\
\hline ACE & 38.1 & 57.8 & 28.8 & 27.4 & 12.4 & 8.0 & 23.2 & 32.7 & 12.9 & 0.27 \\
\hline $\exp [29]$ & 41.8 & 59.7 & 32.4 & 13.2 & 24.5 & 20.5 & 23.2 & 39.0 & 16.0 & 0.22 \\
\hline \multirow[t]{3}{*}{ calc $[8]$} & 67.9 & 75.8 & 42.8 & 41.8 & 19.1 & 16.0 & 24.1 & 41.1 & 17.1 & 0.21 \\
\hline & $\begin{array}{l}C_{11}, \\
\mathrm{GPa}\end{array}$ & $\begin{array}{l}C_{22}, \\
\mathrm{GPa}\end{array}$ & $\begin{array}{l}C_{33}, \\
\mathrm{GPa}\end{array}$ & $\begin{array}{l}C_{44}, \\
\mathrm{GPa}\end{array}$ & $\begin{array}{l}C_{55}, \\
\mathrm{GPa}\end{array}$ & $\begin{array}{l}C_{66}, \\
\text { GPa }\end{array}$ & $\begin{array}{l}B_{\mathrm{R}}, \\
\mathrm{GPa}\end{array}$ & $\begin{array}{l}E_{\mathrm{R}}, \\
\mathrm{GPa}\end{array}$ & $\begin{array}{l}G_{\mathrm{R}}, \\
\mathrm{GPa}\end{array}$ & $v_{\mathrm{R}}$ \\
\hline & \multicolumn{10}{|c|}{ b) } \\
\hline DFT & 79.7 & 37.3 & 71.4 & 7.2 & 53.7 & -0.09 & 25.5 & 41.4 & 16.8 & 0.23 \\
\hline ACE & 75.7 & 35.3 & 72.3 & 6.4 & 48.9 & -0.36 & 31.9 & 49.1 & 19.7 & 0.24 \\
\hline calc $[30]$ & 73 & 37 & 82 & 2 & 53 & 7 & 32 & 54 & 22 & 0.28 \\
\hline
\end{tabular}

На рис. 1 показаны зависимости энергии основного состояния Pnma и $\mathrm{Cmcm}$ фаз SnSe. Видно, что энергетически две фазы достаточно близки друг к другу, а ACEпотенциал точно воспроизводит кривизну референтных кривых, полученных из DFT-расчетов. Обработка кривых уравнением состояния Бирча-Мурнагана приводит к значениям модуля всестороннего сжатия 24.8 и 31.4 ГПа для Pnma и $\mathrm{Cmcm}$ фаз соответственно. Следует отметить, что данные значения, хорошо согласующиеся с представленными выше результатами расчета МВС из упругих констант, удается получить только при условии релаксации атомных позиций при каждом значении объема элементарной ячейки. В отсутствие релаксации получаем значения $\mathrm{MBC}$, практически в 2 раза превышающие референтные.

Фононный спектр рассчитывался с помощью программного пакета Phonopy [31], при этом размер суперьячейки составлял $2 \times 4 \times 4$ и $4 \times 2 \times 4$ для Рпта и $\mathrm{Cmcm}$ соответственно, атомы смещались на $0.01 \AA$ относительно своих положений равновесия. Результаты

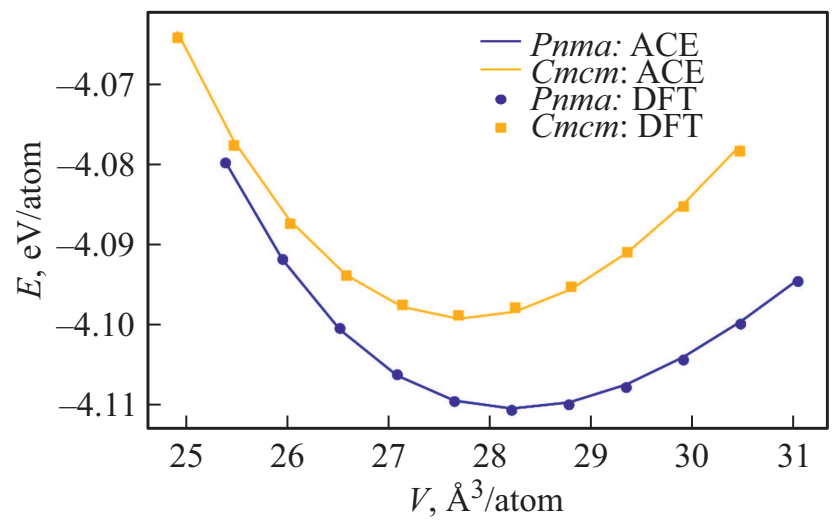

Pис. 1. Зависимость энергии основного состояния Рпma и $\mathrm{Cmcm}$ фаз $\mathrm{SnSe}$ от объема. Здесь и далее на графиках $\mathrm{ACE}$ - расчет в данной работе с помощью АСЕ-потенциала, DFT - расчет в данной работе на основе DFT. расчетов плотности состояний фононов (phDOS) при использовании в качестве вычислителя сил, действующих на атомы, пакета VASP (DFT-расчет) и ACE-потенциала показаны на рис. 2: видно, что разработанный потенциал обеспечивает достоверное описание phDOS во всем интервале энергий для обеих фаз.

В хорошем согласии находятся данные наших расчетов с результатами [4], полученными на основе DFT. Как упоминалось выше, Cmcm-фаза неустойчива при нуле температур, поэтому в случае ее фононного спектра наблюдается некоторое количество мнимых мод. По этой причине далее мы приводим результаты моделирования тепловых и упругих свойств только для Pnma-фазы.

\section{3. Температурные зависимости термодинамических свойств}

Как видно из рис. 3, в целом значения ОКТР SnSe, полученные на основе разработанного АCЕ-потенциала, согласуются с экспериментальными данными, но модель предсказывает несколько более сильное тепловое расширение. Однако заметно также, что данные экспериментов [6] и [32] не вполне согласуются между собой, что указывает на необходимость дополнительных измерений. Значительная неопределенность в экспериментальных значениях свойственна и $\mathrm{MBC} \mathrm{SnSe}$, как можно видеть из рис. 4, где данные экспериментов [33] и [32] показаны в сравнении с результатами расчета в настоящей работе. В целом наша модель адекватно описывает температурную зависимость МВС Рпma-фазы $\mathrm{SnSe}$, при этом ранее подобные расчеты в литературе отсутствовали.

Результаты расчета молярной теплоемкости при постоянном давлении (см. рис. 5) при высоких температурах не прогнозируют резкого возрастания в области температур > $600 \mathrm{~K}$, наблюдаемого в эксперименте [34-36]. По-видимому, этот рост обусловлен приближением к фазовому переходу в $\mathrm{Cmcm-фазу,} \mathrm{когда} \mathrm{резко} \mathrm{возрастает}$ 

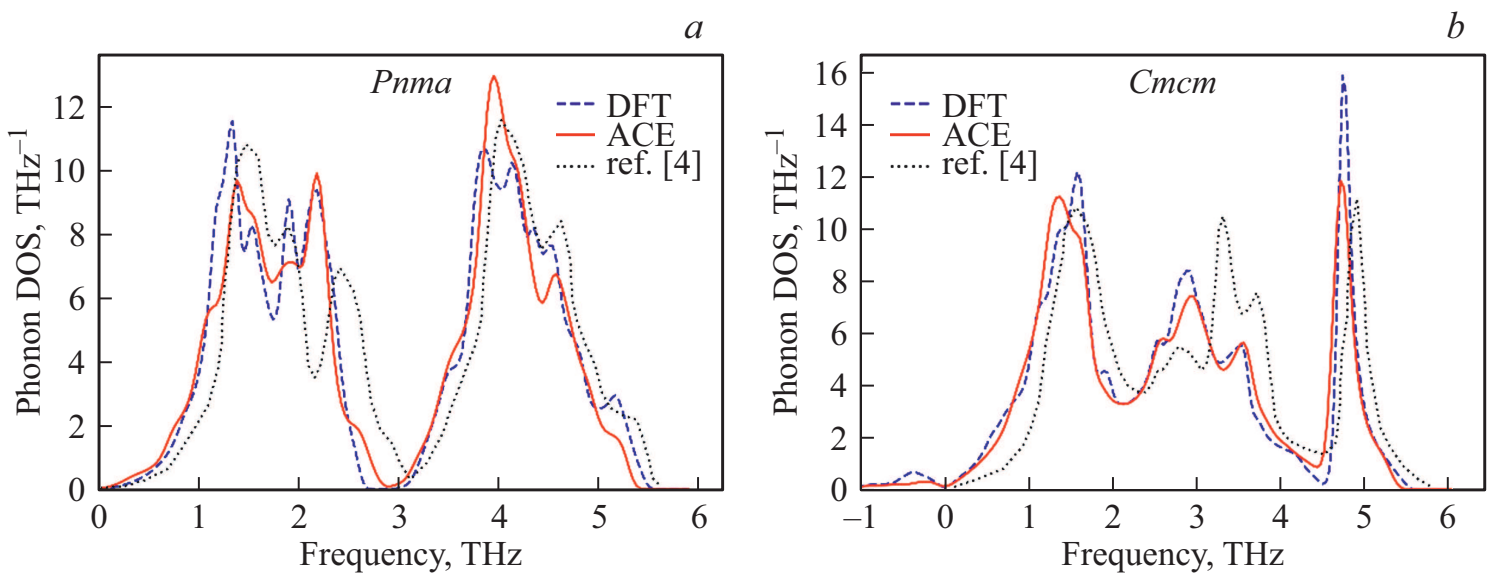

Рис. 2. Плотность фононных состояний $\mathrm{SnSe}$.

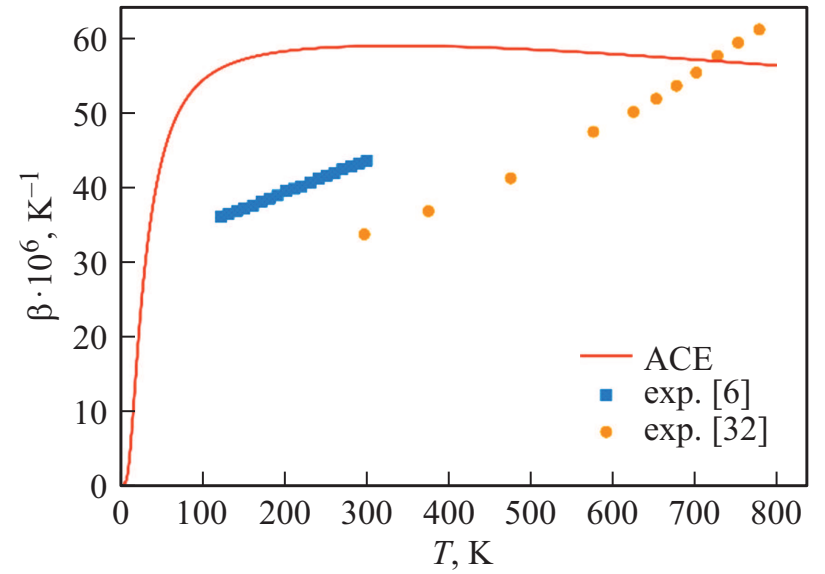

Рис. 3. Температурная зависимость ОКТP SnSe.

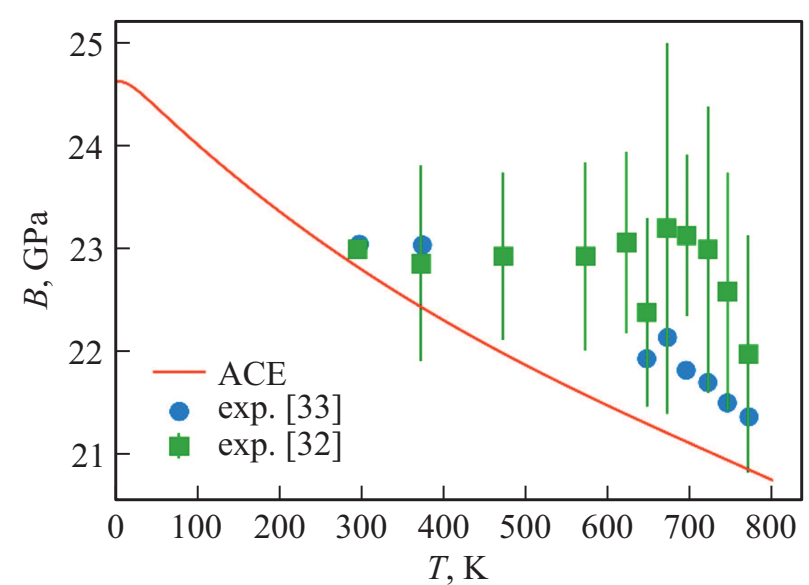

Рис. 4. Температурная зависимость MBC SnSe.

ангармоничность решеточных колебаний, не полностью учитываемая в квазигармоническом приближении. Кроме того, в этой области температур могут образовываться дефекты, например вакансии и междоузлья, даю- щие дополнительный вклад в теплоемкость, который не рассматривается в нашей вычислительной схеме. Также следует отметить, что в области низких температур расчетная кривая находится выше экспериментальных значений, что является следствием погрешности DFTрасчетов фононного спектра, на которых базируется межатомный ACE-потенциал. Данная проблема является достаточно распространенной при DFT-расчетах фононов и обусловливает тот факт, что для точного определения решеточной составляющей теплоемкости (например, для оценки вкладов электронной/магнитной подсистем) необходимо также привлечение модельных подходов на основе приближений Дебая/Эйнштейна [37].

Как упоминалось во введении, в случае $\mathrm{SnSe}$ наблюдается большой разброс значений параметра Грюнайзена, поэтому на рис. 6 мы приводим его значения, полученные на основе формулы $\Gamma=\beta B_{T} V / C_{V}$ (см. Введение) и рассчитанных в настоящей работе значений термодинамических свойств.

Видно, что достаточно высокие значения параметр Грюнайзена принимает при низких температурах, однако после достижения максимума начинает резко убывать

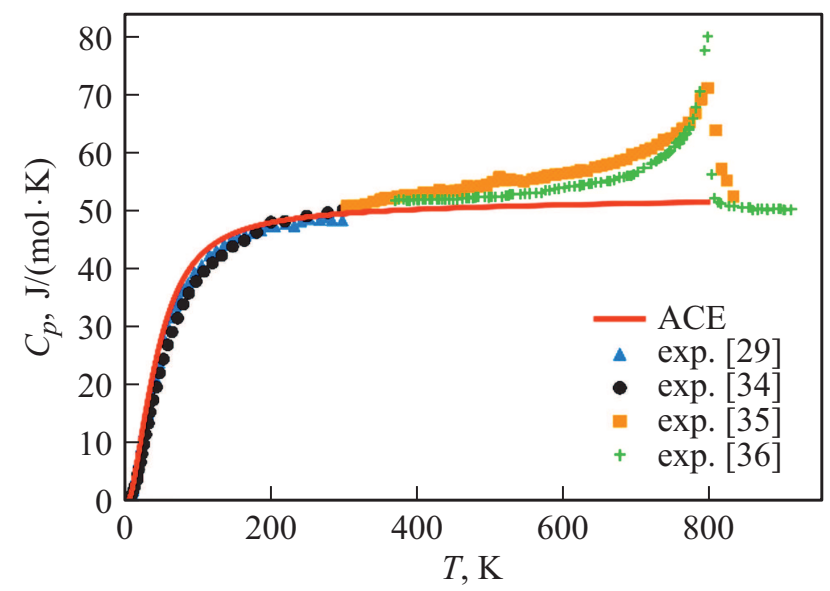

Рис. 5. Температурная зависимость молярной теплоемкости SnSe. 


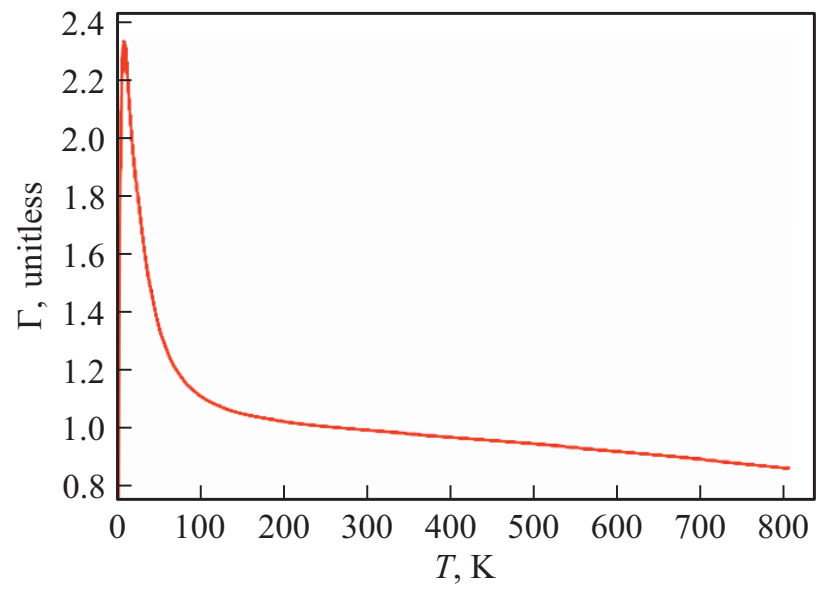

Рис. 6. Температурная зависимость параметра Грюнайзена SnSe.

и в районе $300 \mathrm{~K}$ не превышает единицы, что является весьма малым значением, не свойственным материалам с сильным решеточным ангармонизмом. С другой стороны, данное значение вполне оправданно, учитывая достаточно малые величины ОКТР и MBC SnSe. Причину столь сильного расхождения значения параметра Грюнайзена, следующего из термодинамического анализа и полученного, например, из объемной производной фононных частот, можно понять, если вспомнить о сильной анизотропии теплового расширения $\mathrm{SnSe}$. Так, в работе [8] были получены обобщенные параметры Грюнайзена, соответствующие одноосным деформациям кристаллической структуры. В выражении для ОКТР эти параметры умножаются на постоянные упругой податливости, некоторые из которых в случае $\mathrm{SnSe}$ оказываются отрицательными, в результате чего получается эффект незначительного теплового расширения при достаточно больших значениях параметров Грюнайзена. Иными словами, $\mathrm{SnSe}$ является примером проявления того факта, что различные определения параметра Грюнайзена не всегда эквивалентны друг другу.

\section{4. Заключение}

На основе ряда выполненных первопринципных расчетов для различных кристаллических структур селенида олова для него был получен ACE-потенциал межатомного взаимодействия. Параметры структуры, упругих свойств и фононных спектров, рассчитанные на основе полученного потенциала, находятся в отличном согласии с референтными DFT-данными, что свидетельствует о том, что АСЕ-потенциал может быть использован для корректного атомистического моделирования по крайней мере Pnma- и Cmcm-фаз $\mathrm{SnSe}$. На основе ACEпотенциала вычислены плотности фононных состояний Pnта-фазы при различных объемах. Это позволило выполнить моделирование температурных зависимостей тепловых и упругих свойств Рnта-фазы SnSe в рамках квазигармонического приближения, результаты которого хорошо согласуются с экспериментальными данными. Впервые получено описание температурной зависимости MBC SnSe на основе $a b$ initio расчетов его фононного спектра.

Логичным направлением последующего применения полученного ACE-потенциала является проведение на его основе молекулярно-динамических симуляций $\mathrm{SnSe}$, которые бы позволили детальнее исследовать фазовые превращения в данном материале, а также получить температурные зависимости его свойств с полным учетом ангармонических эффектов. Последнее особенно актуально для высокотемпературной $\mathrm{Cmcm}$-фазы, свойства которой невозможно рассчитать на основе фононного спектра в нуле температур по причине наличия мнимых частот.

\section{Финансирование работы}

Работа выполнена в рамках проекта, финансируемого Министерством науки и высшего образования Российской Федерации (паспорт проекта № 2291-21) и германской службой академических обменов DAAD (№ 57515328) по программе „Михаил Ломоносов““, анализ и интерпретация данных выполнялись при поддержке финансирования в рамках государственного задания Министерства науки и высшего образования Российской Федерации № FEUZ-2020-0020.

\section{Конфликт интересов}

Авторы заявляют, что у них нет конфликта интересов.

\section{Список литературы}

[1] Z.-G. Chen, X. Shi, L. Zhao, J. Zou. Progr. Mater. Sci., 97, 283 (2018).

[2] L.-D. Zhao, C. Chang, G. Tan, M.G. Kanatzidis. Energy Environ. Sci., 9, 3044 (2016).

[3] T. Lanigan-Atkins, S. Yang, J.L. Niedziela, D. Bansal, A.F. May, A.A. Puretzki, J.Y. Lin, D.M. Pajerowski, T. Hong, S. Chi, G. Ehlers, O. Delaire. Nature Commun., 11, 4430 (2020).

[4] Y. Lu, F. Zheng, Y. Yang, F. Zheng, Y. Yang, P. Zhang, D.B. Zhang. Phys. Rev. B, 100, 054304 (2019).

[5] U. Aseginolaza, R. Bianco, L. Monacelli, L. Paulatto, M. Calandra, F. Mauri, A. Bergara, I. Errea. Phys. Rev. Lett., 122, 075901 (2019).

[6] J.S. Kang, H. Wu, M. Li, Y. Hu. Nano Lett., 19, 4941 (2019).

[7] D. Wang, W. He, C. Chang, G. Wang, J. Wang, L.-D. Zhao. J. Mater. Chem. C, 6, 12016 (2018).

[8] G. Liu, J. Zhou, H. Wang. Phys. Chem. Chem. Phys., 19, 15187 (2017).

[9] H. Yu, S. Dai, Y. Chen. Sci. Rep., 6, 26193 (2016).

[10] A. Dewandre, O. Hellman, S. Bhattacharya, A.H. Romero, G.K.H. Madsen, M.J. Verstraete. Phys. Rev. Lett., 117, 276601 (2016).

[11] Y. Xiao, C. Chang, Y. Pei, D. Wu, K. Peng, X. Zhou, S. Gong, J. He, Y. Zhang, Z. Zeng, L.-D. Zhao. Phys. Rev. B, 94, 125203 (2016). 
[12] J.M. Skelton, L.A. Burton, S.C. Parker, A. Walsh, C.E. Kim, A. Soon, J. Buckeridge, A.A. Sokol, C.R.A. Catlow, A. Togo, I. Tanaka. Phys. Rev. Lett., 117, 075502 (2016).

[13] R. Drautz. Phys. Rev. B, 99, 014104 (2019).

[14] Y. Lysogorskiy, C.v.d. Oord, A. Bochkarev, S. Menon, M. Rinaldi, T. Hammerschmidt, M. Mrovec, A. Thompson, G. Csányi, C. Ortner, R. Drautz. npj Comput. Mater., 7, 97 (2021).

[15] R. Golesorkhtabar, P. Pavone, J. Spitaler, P. Puschnig, C. Draxl. Comput., Phys. Commun., 184, 1861 (2013).

[16] S. Plimpton. J. Comp. Phys., 117, 1 (1995).

[17] http://lammps.sandia.gov

[18] G. Kresse, J. Hafner. Phys. Rev. B, 47, 558 (1993).

[19] G. Kresse, G. Furthmüller. Phys. Rev. B, 54, 11169 (1996).

[20] P.E. Blöchl, O. Jepsen, O.K. Andersen. Phys. Rev. B, 49, 16223 (1994).

[21] J. Perdew, K. Burke, M. Ernzerhof. Phys. Rev. Lett., 77, 3865 (1996).

[22] J. Janssen, S. Surendralal, Y. Lysogorskiy, M. Todorova, T. Hickel, R. Drautz, J. Neugebauer. Comput. Mater. Sci., 163, 24 (2019).

[23] A.H. Larsen, J.J. Mortensen, J. Blomqvist, I.E. Castelli, R. Christensen, M. Dułak, J. Friis, M.N. Groves, B. Hammer, C. Hargus, E.D. Hermes, P.C. Jennings, P.B. Jensen, J. Kermode, J.R. Kitchin, E.L. Kolsbjerg, J. Kubal, K. Kaasbjerg, S. Lysgaard, J.B. Maronsson, T. Maxson, T. Olsen, L. Pastewka, A. Peterson, C. Rostgaard, J. Schiøtz, O. Schütt, M. Strange, K.S. Thygesen, T. Vegge, L. Vilhelmsen, M. Walter, Z. Zeng, K.W. Jacobsen. J. Phys.: Condens. Matter, 29, 273002 (2017).

[24] S. Chen, K.F. Cai, W. Zhao. Physica B: Condens. Matter, 407, 4154 (2012).

[25] H.G. Von Schnering, H. Wiedemeier. Z. Kristallogr. Cryst. Mater, 156, 143 (1981).

[26] I.R. Nuriev, A.K. Sharifova. Sov. Phys. Crystallogr., 34, 635 (1989).

[27] A. Jain, S.P. Ong, G. Hautier, W. Chen, W.D. Richards, S. Dacek, S. Cholia, D. Gunter, D. Skinner, G. Ceder, K.A. Persson. APL Mater., 1, 011002 (2013).

[28] R. Hill. Proc. Phys. Soc. (London), 65, 349 (1952).

[29] A. Karunarathne, J.R. Gladden, G. Priyadarshan. ACS Appl. Energy Mater., 1, 6123 (2018).

[30] M. de Jong, W. Chen, T. Angsten, A. Jain, R. Notestine, A. Gamst, M. Sluiter, C.K. Ande, S. vanderZwaag, J.J. Plata, C. Toher, S. Curtarolo, G. Ceder, K.A. Persson, M. Asta. Sci. Data, 2, 150009 (2015).

[31] A. Togo, I. Tanaka. Scr. Mater., 108, 1 (2015).

[32] A. Karunarathne, P. Parajuli, G. Priyadarshan, S. Bhattacharya, R. Rao, P. Wei, Y. Chen, J.R. Gladden, A.M. Rao. Phys. Rev. B, 103, 054108 (2021).

[33] F. Liu, P. Parajuli, R. Rao, P.C. Wei, A. Karunarathne, S. Bhattacharya, R. Podila, J. He, B. Maruyama, G. Priyadarshan, J.R. Gladden, Y.Y. Chen, A.M. Rao. Phys. Rev. B, 98, 224309 (2018).

[34] S.R. Popuri, M. Pollet, R. Decourt, M.L. Viciu, J.W.G. Bos. Appl. Phys. Lett., 110, 253903 (2017).

[35] S. Sassi, C. Candolfi, J.-B. Vaney, V. Ohorodniichuk, P. Masschelein, A. Dauscher, B. Lenoir. Appl. Phys. Lett., 104, 212105 (2014).

[36] C.-H. Lee, M.-H. Ma, W.-H. Li, P.-C. Wei, Y.-Y. Chen, Y. Zhao, J.W. Lynn. Mater. Today Phys., 11, 100171 (2019).

[37] A.N. Filanovich, A.A. Povzner. Physica B, 527, 16 (2017).

\section{Atomistic simulation of the lattice properties of $\mathrm{SnSe}$}

\author{
A.N. Filanovich ${ }^{1}$, Y.V. Lysogorskiy ${ }^{2}$, A.A. Povzner ${ }^{1}$
}

${ }^{1}$ Ural Federal University named after the first President of Russia B.N. Yeltsin,

620002 Ekaterinburg, Russia

2 ICAMS, Ruhr-Universitat Bochum, 44801 Bochum, Germany

\begin{abstract}
Abstract We have performed a set of ab initio calculations of the ground state energy as a function of volume, elastic properties, and phonon spectra of tin selenide in its various crystalline modifications. Based on the obtained data set, the potential of interatomic interaction of $\mathrm{SnSe}$ is constructed using the atomic cluster expansion (ACE) method. The potential is further used to study the temperature dependences of thermal and elastic properties of $\mathrm{SnSe}$ in the framework of quasi-harmonic approximation.
\end{abstract}

